\title{
Multiple Coat Protein Mutations Abolish Recognition of Pepino mosaic potexvirus (PepMV) by the Potato Rx Resistance Gene in Transgenic Tomatoes
}

\author{
Thierry Candresse, ${ }^{1}$ Armelle Marais, ${ }^{1}$ Chantal Faure, ${ }^{1}$ Marie Pierre Dubrana, ${ }^{1}$ Julie Gombert, ${ }^{2}$ and \\ Abdelhafid Bendahmane ${ }^{2}$ \\ ${ }^{1}$ Equipe de Virologie, UMR GD2P, IBVM, INRA and Université Victor Ségalen Bordeaux2, BP81, 33883 Villenave d'Ornon \\ Cedex, France; ${ }^{2}$ INRA-URGV 2, Rue Gaston Crémieux CP 570891057 EVRY Cedex, France
}

Submitted 11 August 2009. Accepted 12 November 2009.

\begin{abstract}
Despite the fact that Pepino mosaic virus (PepMV) and Potato virus $X$ (PVX) share less than $40 \%$ identity in their coat proteins (CP), the known PVX elicitor of $R x$, transgenic tomato (cv. Microtom) plants expressing a functional potato $R x$ resistance gene showed resistance toward PepMV. However, in a low percentage of plants, PepMV accumulation was observed and back inoculation experiments demonstrated that these plants contained resistancebreaking PepMV variants. Sequencing of the $\mathrm{CP}$ gene of these variants showed the accumulation of mutations in the amino acid 41 to 125 region the $C P$, whereas no mutations were observed in the nonevolved isolates. Agroinfiltrationmediated transient expression of the mutant CP demonstrated that they had a greatly attenuated or abolished ability to induce a hypersensitive reaction in $\boldsymbol{R} x$-expressing Nicotiana benthamiana leaves. The transient expression of truncated forms of the PepMV CP allowed the identification of a minimal elicitor domain (amino acids 30 to 136). These results demonstrate that the $R x$-based sensing system is able to recognize the PepMV CP but, contrary to the situation with PVX, for which only two closely spaced resistance-breaking mutations are known, many mutations over a significant stretch of the PepMV CP allow escape from recognition by $R x$.
\end{abstract}

A number of genes and proteins allowing the recognition by plants of pathogen-derived signals have now been identified. The vast majority of these are resistance $(R)$ genes involved in race-specific interactions (Hammond-Kosack and Jones 1997; Ellis et al. 2000; Martin et al. 2003; Chisholm et al. 2006; Bent and Mackey 2007) that participate in the recognition of pathogen avirulence ( $A v r$ ) factors. The $R x$ gene of potato confers racespecific resistance against Potato virus $X$ (PVX) (Cockeram 1970), a member of the genus Potexvirus. At least two orthologs with similar recognition specificity, $R x 1$ and $R x 2$, have been identified, originating from two different wild Solanum spp., Solanum andigena ( $R x 1$ or $R x_{a d g}$, located on potato chromosome XII) (Ritter et al. 1991; Bendahmane et al. 1999) and

Corresponding author: T. Candresse; Telephone : +33 (0) 5571223 89; Fax : +33 (0) 5571223 84; E-mail : tc@ bordeaux.inra.fr

Nucleotide sequences reported in this manuscript have been deposited in GenBank under accession numbers EU730952 to EU730964.

* The $e$-Xtra logo stands for "electronic extra" and indicates that three supplementary figures are published online.
$S$. acaule ( $R x 2$ or $R x_{a c l}$, located on chromosome V) (Ritter et al. 1991; Bendahmane et al. 2000). Potential additional $R x$ genes with similar resistance phenotype and recognition specificity have also been detected in other wild Solanum spp. or in natural hybrids between potato and wild species (Querci et al. 1995). The $R x$ gene belongs to the coil-coil nucleotide-binding site leucine-rich repeat (CC-NBS-LRR) family of plant resistance genes (Bendahmane et al. 1999; Ellis et al. 2000). Among $R$ genes, the phenotype of the resistance afforded by $R x$ is unusual in several ways, so that the term "extreme resistance" has been coined to describe it (Tozzini et al. 1991). Upon inoculation of $R x$ plants by an avirulent PVX isolate, there is no development of a hypersensitive reaction (HR); however, the accumulation of PVX is rapidly arrested so that, in most situations, it is not possible to detect the virus in the inoculated leaves (Tozzini et al. 1991). In addition, work with protoplasts indicated that $R x$ is active at the single-cell level and that virus accumulation is restricted through the induction of a nonspecific antiviral response (Kohm et al. 1993). Transient gene expression experiments indicate, however, that $R x$ is also able to induce an HR reaction but that the antiviral response is epistatic over the HR which, therefore, is not observed under normal infection conditions (Bendahmane et al. 1999). The $R x$ resistance has proved to be highly durable (Jones 1985) and, thus far, a single PVX resistance-breaking isolate, $\mathrm{HB}$, has been identified (Moreira et al. 1980; Querci et al. 1993). Reverse genetics and transient expression assays have demonstrated that the coat protein $(\mathrm{CP})$ is the viral elicitor recognized by $R x$ (Kavanagh et al. 1992; Goulden and Baulcombe 1993) and that its action does not necessitate any other PVX-encoded factor (Bendahmane et al. 1995). Mutational analysis has revealed a major role of the conserved threonine at position 121 of the $\mathrm{CP}$ in the avirulence toward $R x$, because the mutation to a lysine observed in PVX HB completely abolishes the recognition by $R x$ potato genotypes (Goulden and Baulcombe 1993). However, isolates containing this $\mathrm{T} 121 \mathrm{~K}$ mutation alone are severely affected in their infectivity and in their ability to systemically invade susceptible potato plants. A second mutation, also observed in PVX HB, K127R contributes to the restoration of the fitness of variants containing the $\mathrm{T} 121 \mathrm{~K}$ resistance-breaking mutation (Goulden and Baulcombe 1993).

Pepino mosaic virus (PepMV) (Jones et al. 1980) is a potexvirus able to infect a large range of solanaceous crops. Initially observed in South America in the late 1970s in pepino (S. muricatum), it was rediscovered in the Netherlands in the late 1990s in greenhouse-grown tomato crops (van der Vlugt et al. 2000) and was rapidly observed in this crop in a number of European 
countries, including the United Kingdom (Mumford and Metcalfe 2001), Belgium (Hanssen et al. 2008), Germany, France (Cotillon et al. 2002), Spain (Aguilar et al. 2002), and Italy (Salomone and Roggero 2002). It has also been detected in Canada (French et al. 2001; Fergusson and Shipp 2002) and in the United States (French et al. 2001; Maroon-Lango et al. 2005). It is considered to be an important emerging pathogen in tomato. Although PepMV has apparently never been observed on potato, it has the potential to infect this host and is considered to be a potential threat to potato crops (Jones et al. 1980; Martin and Mouserion 2002). PepMV is only distantly related to PVX and their CP share less than $40 \%$ amino acid sequence identity, while only limited variability has so far been detected by analyzing the CP of PepMV isolates (average divergence 3.7\% for over $100 \mathrm{CP}$ sequences present in GenBank) (Mumford and Metcalfe 2001; Aguilar et al. 2002; Cotillon et al. 2002; Lopez et al. 2005; Maroon-Lango et al. 2005; Hanssen et al. 2008).

Recent results (Baurès et al. 2008) have shown that the $R x$ gene confers resistance not only against PVX but also against other potexviruses. In an effort to identify in solanaceous crops new resistance sources against PepMV, the potential activity of the $R x$ gene against PepMV was evaluated in $R x$-transgenic tomato plants. The results obtained indicate that $R x$ is active against PepMV, despite the wide divergence of its $\mathrm{CP}$ from that of PVX; but that, contrary to the situation observed with PVX, the resistance afforded is unstable, due to the accumulation of mutations in the PepMV CP.

\section{RESULTS}

\section{Resistance to PepMV}

of transgenic Microtom tomato expressing $R \boldsymbol{x}$ resistance.

The resistance to PepMV infection of transgenic tomato plants of cv. Microtom (Meissner et al. 1997) expressing the $R x$ gene under its native promoter was evaluated during this work. These plants were obtained by Agrobacterium-mediated transformation using the same construct that was used to obtain $R x$ resistant transgenic potato and tobacco (Nicotiana tabacum and N. benthamiana) (Bendahmane et al. 1999; Baurès et al. 2008). The transgenic $R x$ Microtom plants obtained showed a complete $R x$ phenotype, in that they are fully resistant against avirulent PVX isolates (but without showing an HR unless the $\mathrm{CP}$ is expressed through agroinfiltration) (Chapman et al. 1992) and are susceptible toward virulent PVX isolates or mutants (Baurès 2008) (Supplementary Fig. 1).

The $R x$-expressing tomato and control, nontransgenic Microtom plants were inoculated with the PV-0632 PepMV isolate. In general, no obvious infection symptoms could be observed in any of the plants, on either the inoculated or noninoculated parts. However, high PepMV enzyme-linked immunosorbent assay (ELISA) readings or tissue immunoprinting assays performed 2 weeks postinoculation indicated that $100 \%$ of the wild-type plants were systemically infected by PepMV (Table 1), indicating that PepMV infection is essentially symptomless in Microtom tomato. Occasionally, some rugosity could be noticed on the leaves of the infected wild-type plants compared with uninoculated controls but this subtle symptom could not be used as a reliable infection indicator. Therefore, immunoassays were used throughout this work to evaluate the infection status of the various plants.

Overall, in four independent inoculation experiments, $100 \%$ of the wild-type tomato plants became infected with the PV0632 isolate of PepMV. Using a lower number of plants and experiments, a similar result was obtained using three other pepino or tomato PepMV isolates (PV-0554, PV-0751, and 1907). For the original pepino isolate of PepMV (PV-0554), these results are consistent with the initial report of symptomless PepMV infection of tomato (Jones et al. 1980). On the other hand, the tomato isolates are known to induce symptoms in greenhouse-grown commercial tomato (van der Vlugt et al. 2000), hinting at an original behavior of cv. Microtom under our assay conditions.

Contrary to the results obtained in the wild-type Microtom plants, $R x$-expressing transgenics showed substantial resistance to PepMV PV-0632. Out of a total of 22 inoculated plants in four independent experiments, only 2 plants in experiment number 2 developed late ( $>1$ month postinoculation) necrotic symptoms. ELISA assays performed on all inoculated $R x$ transgenics showed optical density (OD) values above those of control mock-inoculated plants for only those two symptomatic plants (Table 1). Therefore, the overall rate of infection of the $R x$ transgenics by the PepMV PV-0632 isolate was $9 \%$. Using a smaller number of plants, the resistance of the $R x$ transgenics to PepMV was confirmed for all three other isolates tested, with only 0\% (PepMV PV-0751) to $14.3 \%$ of plants becoming infected, depending on the isolate used (Table 1). Contrary to the situation observed with the wild-type plants in which PepMV was always asymptomatic, PepMV infection in $R x$ transgenics was accompanied by the usually late development of symptoms, including chlorotic or necrotic spots, dwarfing of the plants, or wilting due to the spread of the necrosis observed (Supplementary Fig. 2B, C, and D).

Inoculation experiments were also performed on a mutant $\mathrm{Rx}$ transgenic line, $\mathrm{Rx} 1179$, in which the Rx-mediated resistance is still effective but in which the Rx-mediated HR against some PVX variants is partially compromised (T. Candresse, M.

Table 1. Resistance of transgenic tomato plants (cv. Microtom) expressing the Rx resistance gene to infection by various Pepino mosaic virus (PepMV) isolates

\begin{tabular}{|c|c|c|c|c|c|c|}
\hline \multirow[b]{2}{*}{ PepMV isolate } & \multirow[b]{2}{*}{ Microtom line ${ }^{b}$} & \multicolumn{4}{|c|}{ Infected plant/inoculated plants ${ }^{\mathrm{a}}$} & \multirow[b]{2}{*}{ Total no. $(\%)^{\mathrm{c}}$} \\
\hline & & Exp. 1 & Exp. 2 & Exp. 3 & Exp. 4 & \\
\hline \multirow[t]{3}{*}{ PV-0632 } & WT & $4 / 4$ & $5 / 5$ & $4 / 4$ & $10 / 10$ & $23 / 23(100)$ \\
\hline & $R x$ & $0 / 4$ & $2 / 5$ & $0 / 3$ & $0 / 10$ & $2 / 22(9)$ \\
\hline & $R \times 1179$ & $1 / 4$ & $3 / 5$ & $1 / 4$ & $\mathrm{nt}$ & $5 / 13(38)$ \\
\hline \multirow[t]{2}{*}{ PV-0554 } & WT & $\mathrm{nt}$ & $\mathrm{nt}$ & $4 / 4$ & $10 / 10$ & $14 / 14(100)$ \\
\hline & $R x$ & $\mathrm{nt}$ & $\mathrm{nt}$ & $1 / 3$ & $0 / 10$ & $1 / 13(8)$ \\
\hline \multirow[t]{2}{*}{ PV-0751 } & WT & $\mathrm{nt}$ & $\mathrm{nt}$ & $4 / 4$ & $10 / 10$ & 14/14 (100) \\
\hline & $R x$ & nt & nt & $0 / 3$ & $0 / 10$ & $0 / 13(0)$ \\
\hline \multirow[t]{2}{*}{1907} & WT & $\mathrm{nt}$ & $\mathrm{nt}$ & $4 / 4$ & $10 / 10$ & $14 / 14(100)$ \\
\hline & $R x$ & $\mathrm{nt}$ & $\mathrm{nt}$ & $2 / 4$ & $0 / 10$ & $2 / 14$ (14) \\
\hline
\end{tabular}

${ }^{a}$ For each independent experiment, the number of infected plants (as evaluated by enzyme-linked immunosorbent assay or tissue immunoprinting assays) is indicated over the total of inoculated plants; nt: not tested.

${ }^{\mathrm{b}}$ Tomato (cv. Microtom) inoculated with the PepMV isolates. WT: wild type (nontransgenic control plants); $R x$ : transgenic plants expressing the $R x$ gene; $R x$ 1179: a mutated line derived from the $R x$-expressing line.

${ }^{\mathrm{c}}$ Total number of infected plants is indicated over the total number of inoculated plants, and percentage of infected plants is indicated in parentheses. 
P. Dubrana, and A. Bendahmane unpublished data). Upon inoculation of this line with PepMV isolate PV-0632, results essentially similar to those obtained with the $R x$-transgenic line were observed, with the only exception that the development of PepMV infection accompanied by severe symptoms was observed in a higher proportion of plants (38\%) (Table 1). Taken together, the above results indicate that the Microtom tomato cultivar is fully susceptible to PepMV infection but that $R x$ expressing transgenic plants show resistance to all PepMV isolates tested. However, this resistance is not complete with three of the four PepMV isolates used, with infection symptoms and viral accumulation developing in a low percentage of plants.

\section{Development of PepMV infection \\ in $\boldsymbol{R} x$ transgenics corresponds to the selection of resistance-breaking variants.}

Two hypotheses could be proposed to account for the observed infection of a low percentage of $R x$ transgenics. The first one is that the resistance conferred by the $R x$ transgene could be only partial, allowing the virus to sometimes overcome it. The second possibility is that the few cases of viral accumulation observed correspond to the selection of resistancebreaking variants. In order to discriminate between these two possibilities, back inoculations to wild-type and $R x$-transgenic tomato were performed from a number of the $R x$ transgenics that had developed PepMV infection upon inoculation with the PV-0632 isolate. In all cases tested and regardless of whether the initial infection had occurred in the original $R x$ transgenic line or in the mutated $R x 1179$ line, a high rate of infection of the back-inoculated $R x$ transgenics was observed (Table 2), indicating that the viral isolate present had acquired the ability to efficiently infect tomato plants expressing the $R x$ gene. In only one case, an isolate originally selected on line 1179 infected only one of the three inoculated $R x$ plants. However, upon further propagation on $R x$ transgenics, it readily infected all three tested plants (Table 2). Thus, the overall rate of infection of $R x$ plants for variants initially selected on $R x$ expressing plants was $88 \%$ (Table 1), approximately 10 -fold the rate observed for the parent PV-0632 isolate.

As observed on the initially infected plants, $R x$ plants backinoculated with the resistance-breaking variants developed various symptoms, including stunting, epinasty, chlorotic or

Table 2. Evaluation of the $R x$ resistance-breaking properties of variants of Pepino mosaic virus (PepMV) isolate PV-0632 selected on $R x$-transgenic tomato

\begin{tabular}{lcc}
\hline & \multicolumn{2}{c}{ No. infected/inoculated $(\boldsymbol{\%})^{\mathbf{a}}$} \\
\cline { 2 - 3 } Isolate PV-0632, variant no. & Microtom WT & Microtom $\boldsymbol{R} \boldsymbol{x}$ \\
\hline WT $^{\mathrm{b}}$ & $23 / 23(100)$ & $2 / 22(9)$ \\
Selected on Microtom $R x$ 1179 & $4 / 4$ & $1 / 3$ \\
Pre-22 & $3 / 3$ & $3 / 3$ \\
$1179-1$ & $3 / 3$ & $3 / 3$ \\
$1179-4$ & $3 / 3$ & $3 / 3$ \\
$1179-5$ & $3 / 3$ & $3 / 3$ \\
$1179-15-12$ & & \\
Selected on Microtom $R x$ & $3 / 3$ & $2 / 3$ \\
8 & $3 / 3$ & $3 / 3$ \\
10 & $4 / 4$ & $3 / 3$ \\
$22^{\mathrm{c}}$ & $26 / 26(100)$ & $21 / 24(88)$ \\
Total selected variants & $0 / 11$ & $0 / 9$ \\
Mock inoculated & & \\
\hline
\end{tabular}

${ }^{a}$ Number of infected plants (as evaluated by enzyme-linked immunosorbent assay or tissue immunoprinting assays) is indicated over the total of inoculated plants. When appropriate, the percentage of infected plants is indicated in parentheses.

${ }^{\mathrm{b}}$ Wild-type (unselected) PV-0632 isolate.

c Variant 22 was obtained by passaging variant Pre-22, obtained on the line $R x 1179$ on $R x$ plants. necrotic spots, and spreading necrosis finally causing the death of the plants. In contrast, parallel infection of wild-type Microtom by the resistance-breaking variants remained asymptomatic, indicating that the symptomatology observed on the $R x$ plants is $R x$ mediated.

Back-inoculation experiments performed from a more limited number of $R x$ plants infected with isolates PV-0554 and 1907 yielded essentially similar results (results not shown), indicating that, in the case of these isolates also, development of viral infection on $R x$ plants was associated with the emergence of resistance-breaking variants.

\section{Analysis of the sequence of the $\mathrm{CP}$ gene of $R x$ resistance-breaking variants derived from the PepMV PV-0632 isolate.}

The CP is known to be the viral elicitor recognized by $R x$ potato plants in the case of PVX, and transient expression assays have shown that expression of the PVX CP alone, independently of any other viral factor, is sufficient to induce the $R x$-mediated responses (Goulden et al. 1993; Bendahmane et al. 1995, 1999). The CP of PepMV shows less than $40 \%$ identity with that of PVX but we postulated that the PepMV CP is likely to be the viral determinant recognized by $R x$ and to accumulate mutations in the case of the $R x$-breaking variants described above. Therefore, the $\mathrm{CP}$ gene was amplified by polymerase chain reaction (PCR) from wild-type PV-0632 as well as from resistance-breaking variants derived from it. PV0632 turned out to be very closely related to the US2 isolate (Maroon-Lango et al. 2005). The wild-type sequence was determined in four independent experiments, using different passages in wild-type Microtom, to provide an estimation of the natural variability of the isolate and of the mutations that could be introduced by the PCR procedure. Only two noncoding mutations were observed (only once for each mutation) in the four PV-0632 CP gene sequences determined (Table 3), confirming the known limited variability of PepMV CP (Mumford and Metcalfe 2001; Aguilar et al. 2002; Cotillon et al. 2002; Lopez et al. 2005; Maroon-Lango et al. 2005).

For all evolved variants, the $\mathrm{CP}$ gene sequence was determined from samples taken from $R x$ transgenics. In most cases (five variants), the sequence was determined twice, once from the initially infected $R x$-expressing plant and once after backinoculation to $R x$ plants to confirm the resistance-breaking properties. For two other isolates (nos. 8 and 22), the CP sequence was determined only once, after the confirmatory inoculation on $R x$ plants (Table 3). For some resistance-breaking variants, several sequence variants were observed. In total, $\mathrm{CP}$ gene sequences were determined for seven resistance-breaking variants, yielding a total of 11 variant sequences. All of them showed from two to four point mutations compared with the wild-type isolate, while 10 of 11 showed a C537T noncoding mutation also observed in one of the wild-type PV-0632 sequences. With the exception of one variant (number 1179-1512), sequences determined for different passages were identical, confirming their validity. In the case of 1179-15-12, two initially observed mutations, C314T and G337A (amino acids P105L and A113T, respectively), were not observed upon propagation in $R x$ plants but a new coding mutation (C286A, amino acid Q96K) was observed.

Contrary to the situation observed when comparing the four wild-type CP sequences, all sequences from the resistancebreaking variants showed coding mutations, resulting in one to three amino acid changes in the encoded protein (Table 3). One of the variants (number 1179-15-12) showed a coding, T197C mutation (I66T in the CP amino acid sequence) which reverts the sequence of the PV-0632 isolate to that observed at this position in all other known PepMV isolates (Table 3). 
With the additional exception of the C212T mutation (A71V) in sequences derived from variant $1179-4$, and which is also present in the wild-type PepMV PV-0554 isolate, all other mutations observed were specific to the resistance-breaking variants. Finally, it should be mentioned that, with the exception of the K41R mutation observed in variant 22, all nine coding mutations that are strictly specific to resistance-breaking variants affected a relatively short region of the $\mathrm{CP}$, between amino acid positions 78 and 125 (Fig. 1), which represents only approximately $20 \%$ of the total CP size. Interestingly, in a multiple alignment of the PepMV-PVX CP, the $R x$-breaking mutation at codon 121 of the PVX CP corresponds to amino acid 122 of the PepMV CP and, therefore, falls in the region accumulating the mutations in the PepMV variants (Fig. 1).
Mutations observed in the CP gene

of the $R x$ resistance-breaking variants abolish or strongly affect the ability of the PepMV CP to induce an $R x$-meditated HR in a transient $N$. benthamiana assay.

In order to validate the hypothesis that the mutations observed in the CP gene of the $R x$-breaking variants are indeed associated with resistance breaking, their effect on the elicitor activity of the PepMV CP was evaluated. The wild-type PV-0632 CP gene and the mutant $\mathrm{CP}$ genes where inserted between the $35 \mathrm{~S}$ promoter and terminator of the Agrobacterium binary vector pBin61 (Bendahmane et al. 2000). The construct expressing the wild-type $\mathrm{CP}$ was first transiently expressed by agroinfiltration of leaves of wild-type or of $R x$-expressing transgenic $N$. benthamiana plants. The expression of the wild-type PepMV CP in-

Table 3. Mutations identified in the coat protein gene (CP) of Rx resistance-breaking variants of Pepino mosaic virus (PepMV) isolate PV-0632 and loss of $R x$-mediated hypersensitive response (HR) induction activity associated with these mutations

\begin{tabular}{|c|c|c|c|c|c|c|}
\hline PepMV variant & $\begin{array}{l}\text { Nucleotide mutations } \\
\text { in CP gene }{ }^{\mathrm{a}}\end{array}$ & $\begin{array}{l}\text { Amino acid mutations } \\
\text { in } \mathbf{C P}^{\mathbf{a}}\end{array}$ & $\begin{array}{c}\text { No. of } \\
\text { mutations }\end{array}$ & $\begin{array}{l}\text { No. of coding } \\
\text { mutations }\end{array}$ & $\begin{array}{l}\text { HR induction in } \\
\text { Rx Nicotiana } \\
\text { benthamiana }^{\text {b }}\end{array}$ & $\begin{array}{l}\text { HR induction in } \\
\quad R x \text { tomato }\end{array}$ \\
\hline WT PV-0632c & $\mathrm{T}^{2} 1 \mathrm{~A}^{\mathrm{d}}, C 537 T^{\mathrm{d}}$ & $\ldots$ & $0.5^{\mathrm{e}}$ & 0 & + & + \\
\hline 22 & A122G, G296A & K41R, G99D & 2 & 2 & - & - \\
\hline 8 & $G 232 A, C 537 T$ & A78T & 2 & 1 & $\mathrm{nt}$ & $\mathrm{nt}$ \\
\hline \multirow[t]{2}{*}{$1179-15-12$} & $T 197 C^{\mathrm{f}}, \mathrm{C} 314 \mathrm{~T}, C 537 T$ & $I 66 T^{\mathrm{f}}, \mathrm{P} 105 \mathrm{~L}$ & 3 & 2 & \pm & \pm \\
\hline & $T 197 C^{\mathrm{f}}, \mathrm{G} 337 \mathrm{~A}, C 537 T$ & $I 66 T^{\mathrm{f}}, \mathrm{A} 113 \mathrm{~T}$ & 3 & 2 & nt & nt \\
\hline \multirow{3}{*}{$\begin{array}{l}1179-15-12 \\
\text { passaged on } R x \\
1179-4^{\mathrm{g}}\end{array}$} & $T 197 C^{\mathrm{f}}, \mathrm{C} 286 \mathrm{~A}, C 537 T$ & $I 66 T^{\mathrm{f}}, \mathrm{Q} 96 \mathrm{~K}$ & 3 & 2 & $\mathrm{nt}$ & $\mathrm{nt}$ \\
\hline & $\mathrm{C}^{2} 12 \mathrm{~T}^{\mathrm{h}}, \mathrm{G} 340 \mathrm{~A}, C 537 T$ & $\mathrm{~A} 71 \mathrm{~V}^{\mathrm{h}}, \mathrm{A} 114 \mathrm{~T}$ & 3 & 2 & nt & $\mathrm{nt}$ \\
\hline & $\mathrm{C} 212 \mathrm{~T}^{\mathrm{h}}, \mathrm{C} 234 \mathrm{~A}, \mathrm{G} 340 \mathrm{~A}, \mathrm{C} 537 \mathrm{~T}$ & $\mathrm{~A} 71 \mathrm{~V}^{\mathrm{h}}, \mathrm{A} 114 \mathrm{~T}$ & 4 & 2 & - & \pm \\
\hline $1179-1^{\mathrm{g}}$ & $G 232 A, C 537 T$ & A78T & 2 & 1 & \pm & \pm \\
\hline & G295A, C537T & G99S & 2 & 1 & - & - \\
\hline \multirow{2}{*}{$R \times 10^{\mathrm{g}}$} & C299A, A374G, C537T & A100D, Q125R & 3 & 2 & - & - \\
\hline & G232A, C299A, A374G, C537T & $A 78 T, \mathrm{~A} 100 \mathrm{D}, \mathrm{Q} 125 \mathrm{R}$ & 4 & 3 & - & - \\
\hline
\end{tabular}

${ }^{a}$ For some resistance-breaking variants, two sequence variants were identified. Mutations observed in several variants are indicated in italics.

${ }^{\mathrm{b}}+$ indicates that an HR was observed upon transient expression by agroinfiltration of the relevant CP form in the leaves of transgenic $R x$-expressing Nicotiana benthamiana; - indicates that no reaction was observed; nt: not tested; \pm denotes an incomplete HR phenotype, with a greenish-brown phenotype of the infiltrated areas.

${ }^{c}$ Mutations observed in four independently obtained PepMV wild-type (WT) PV-0632 sequences.

${ }^{\mathrm{d}}$ Mutations indicated were each observed in only one of the four sequences determined.

e Average value is provided, corresponding to a total of two mutations observed for four sequences determined.

${ }^{\mathrm{f}}$ Mutation indicated reverts the PV-0632 sequence to that of all other known PepMV isolates.

${ }^{\mathrm{g}}$ Identical sequences were obtained for the initial resistance-breaking variant and for the variant passaged once more on $R x$-expressing plants.

${ }^{\mathrm{h}}$ Mutation indicated is observed in at least one WT PepMV isolate (PV-0554) which is susceptible to the $R x$ resistance.

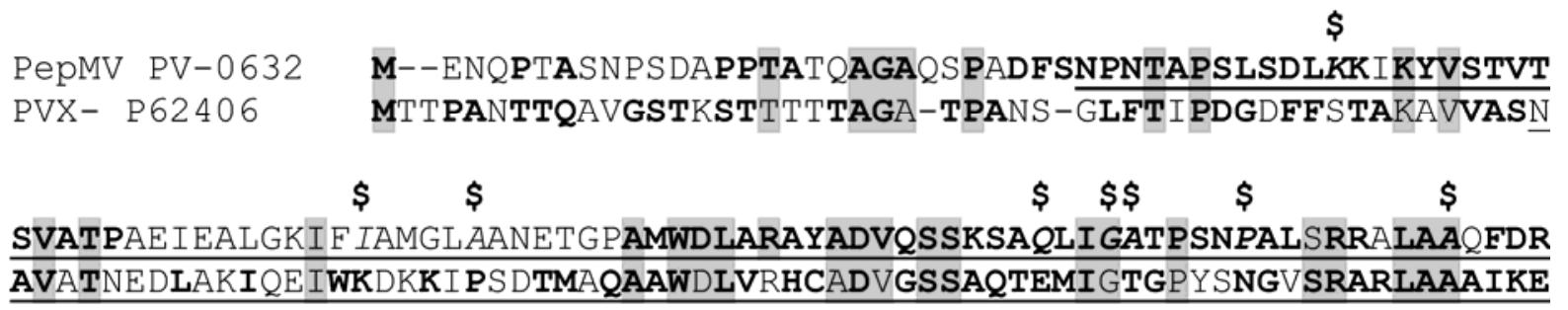

$\$$

INITPRQFCMYFAKIVWNILLDSNVPPANWAKLGYQEDTKFAAFDFFDGVTNPASLQPADGLIRQPNE V-CTLRQFC KKYAPVVWNWMLTNNSPPANWQAQGFKPEHKFAAFDFFDGVTNPAAIT PKEGLMRPPSE 8

\section{KELAAHSVAKYGALARQKISTGNYITTLGEVTRGHMGGANTMYAID-APPEL AEMNAAQTAAFVKITKARAQSNDFASLDAAVTRGRITGTTVAEAVVSLPPP-}

Fig. 1. Sequence alignment of the coat proteins (CP) of Pepino mosaic virus (PepMV) and of Potato virus X (PVX) showing mutations identified in the PepMV Rx resistance-breaking isolates. The alignment was prepared using the PepMV PV-0632 CP sequence and a representative sequences for PVX (accession number indicated). Amino acids fully conserved between all sequenced isolates of a given virus are indicated in bold while amino acids conserved in the alignment between the two viruses are shaded in gray. Amino acids affected by mutations observed in Rx-breaking isolates of PVX or of PepMV (this work) are indicated in italics in the sequences and are noted by \$ (PepMV mutations, above PepMV sequence) and \% (PVX mutations, below PVX sequence) in the multiple alignment. The minimal elicitors (PVX [Baurès et al. 2008] and PepMV [this work]) are underlined in the respective viral sequences. 
duced an HR in the infiltrated part of the $R x$-expressing plants but not in the wild-type plants (Table 3; Supplementary Fig. 3), confirming that similar to the situation reported for PVX (Bendahmane et al. 1999) and for other potexviruses targeted by Rx (Baurès et al. 2008), the PepMV CP is recognized by the $R x$ based sensor, eliciting an $R x$-mediated HR. Transient expression of mutated forms of the $\mathrm{CP}$ isolated from $R x$-breaking variants showed that their ability to induce an $R x$-mediated HR was abolished or very severely affected (Table 3 ). Indeed, for most mutant $\mathrm{CP}$, the infiltrated wild-type and $R x$-expressing leaves were indistinguishable from leaves infiltrated with bacteria harboring the empty pBin61 vector whereas, for two mutants (nos. 117915-12 and 1179-1), the infiltrated zones on $R x$ leaves had a distinct, greenish-brown appearance, still clearly different from the fully necrotic area induced by expression of the wild-type PepMV CP. Control Western blotting experiments demonstrated that the mutated $\mathrm{CP}$ accumulated to levels similar to that observed for the wild-type $\mathrm{CP}$ in the agroinfiltrated leaves (result not shown), indicating that reduction or abolition of the eliciting activity was not due to a lack of expression or to an instability of the mutated CP.

Similar experiments were also performed in leaves of transgenic $R x$-expressing tomato plants. Although infiltration of tomato leaves is significantly less efficient, results largely similar to those observed in $N$. benthamiana were observed, with the only exception that an additional mutant $\mathrm{CP}$, derived from variant 1179-4, gave an incomplete HR phenotype.

\section{Identification of a minimal elicitor region in the PepMV CP.}

Five N-terminally or C-terminally truncated forms of the wild-type PepMV CP were also evaluated in the same transient expression assay for their ability to trigger an $R x$-mediated HR (Fig. 2). Deletion of the first 30 amino acids of the CP or deletion of a large C-terminal portion up to amino acid 136 failed to abolish the HR-inducing activity. On the contrary, CP forms deleted of their first 88 amino acids failed to induce any HR (Fig. 2). In control Western blot experiments, the accumulation of the complete $\mathrm{CP}$ and of the form truncated of the first 30 amino acids could be verified but no signal was obtained from any of the other deleted forms evaluated (results not shown).

\section{DISCUSSION}

The $R x$-transgenic tomato plants used in this work express a complete $R x$ resistance phenotype when confronted with PVX
(Baurès 2008; A. Bendahmane, unpublished). Such an observation is not surprising, in view of the fact that the $R x$ construct used to obtain these plants also conferred a complete $R x$ phenotype to transgenic potato, $N$. benthamiana, and $N$. tabacum plants (Bendahmane et al. 1999; Baurès et al. 2008) and of the frequent demonstration that $R$ genes isolated from solanaceous plants retain their effectiveness when transferred to other plants in the same botanical family (Rommes et al. 1995; Whitham et al. 1996; Tai et al. 1999). The results reported here show, more remarkably, that, despite extensive sequence divergence between the CP of PVX and PepMV (Fig. 1), the $R x$ gene allows recognition of the PepMV CP and confers resistance against this virus in transgenic tomato plants. This observation was verified here with four isolates belonging either to the common or US2 strains of PepMV and parallels the recent report by Baurès and associates (2008) of $R x$-mediated resistance against two other potexviruses, White clover mosaic virus (WCIMV) and Narcissus mosaic virus (NMV), in transgenic $R x$-expressing $N$. benthamiana plants. It remains to be evaluated whether the same effect of $R x$ will be also observed against other divergent strains of PepMV.

Similar to the CP of PVX, WClMV, NMV, and Cymbidium mosaic virus (Baurès et al. 2008) the $\mathrm{CP}$ of PepMV induces an $R x$-dependent HR in transgenic $N$. benthamiana or tomato leaves. The experiments involving the transient expression of the truncated forms of the PepMV CP allow the delineation of a 107-amino-acid-long, minimal elicitor which corresponds to positions 30 to 136 of the PepMV CP. Several reasons could explain the fact that, despite its HR-inducing activity, this minimal elicitor could not be detected in infiltrated leaves by Western blotting, one possibility being that the antiserum used could target epitopes located in the deleted parts. The identification of this minimal elicitor is in agreement with the previous observation that a 90-amino-acid fragment limited between amino acids 50 and 139 of the PVX CP (equivalent to positions 50 to 140 of the PepMV CP) (Fig. 1) is able to elicit the $R x$ mediated response (Baurès et al. 2008) and with the observed clustering of a majority of the mutations observed in the $R x$ breaking variants within a region corresponding to amino acids 78 to 125 of the PepMV CP. Remarkably, this shorter region is almost absolutely conserved between all natural PepMV isolates sequenced to date, with only two of 112 PepMV CP sequences in GenBank showing one or two point mutations (not shown). In this short region, the CP of PepMV and of PVX show only $36 \%$ identity and the longest blocks of identity are three amino acids long (Fig. 1), indicating a high level of flexi-

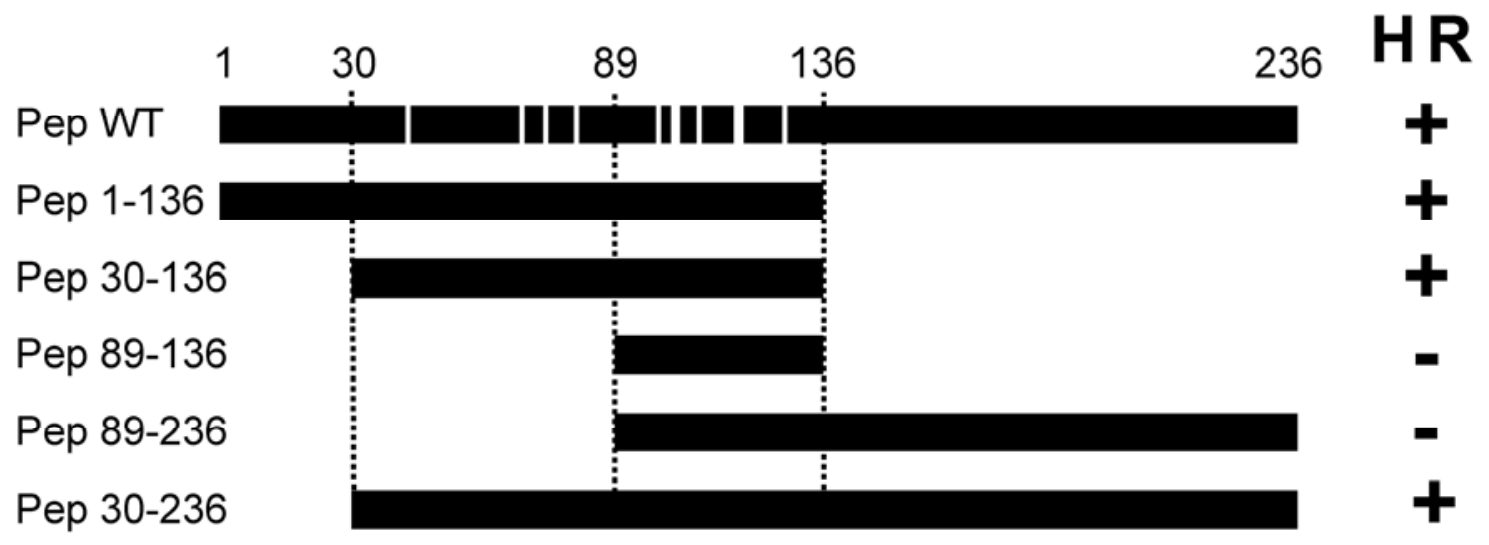

Fig. 2. Schematic representation and hypersensitive reaction (HR) inducing activity in transgenic Rx-expressing Nicotiana benthamiana plants of the full length and of truncated version of the Pepino mosaic virus (PepMV) capsid protein. The full-length wild-type PepMV PV-0632 is depicted at the top (Pep WT) and the N- and C-terminally truncated forms are depicted below. The amino acid positions of the deletions are indicated above the WT coat protein. White bars in the WT CP mark the position of the mutations identified in the resistance breaking isolates. The HR inducing activity upon agroinfiltrationmediated transient expression in $R x$-expressing $N$. benthamiana leaves is indicated on the right; + = induction of an HR; $-=$ no HR-inducing activity. 
bility in the recognition pattern of the $R x$-based sensor and suggesting that a conserved tridimensional structure rather than a linear amino acid sequence might constitute the recognition target. The possibility of recognition of such a tridimensional structure has previously been raised (Chapman et al. 1992; Goulden et al. 1993; Baurès et al. 2008), following secondary structure predictions which indicated that the key Threonine 121 of the PVX CP is likely to be buried (Baratova et al. 1992a and b) and that mutations at this position could potentially induce conformational changes in the CP (Querci et al. 1993). The observations that other potexviruses are able to induce $R x$-mediated HR in transgenic $N$. benthamiana (Baurès et al. 2008) or that artificially mutated versions of $R x$ are able to recognize the distantly related Poplar mosaic virus (Farnham and Baulcombe 2006) provide further support for this hypothesis.

Therefore, the $R x$ gene product could be considered as participating to a molecular sensor recognizing conserved structural elements in the $\mathrm{CP}$ of potexviruses and possibly other flexuous viruses sharing $\mathrm{CP}$ of common phylogenetic ancestry (Dolja et al. 1991). Such elements could be envisioned as occupying an intermediate position between the widely conserved pathogen-associated molecular patterns (PAMP) recognized by receptor-like kinases playing a key role in innate immunity (Brunner et al. 2002; Parker 2003; Boller and Felix 2009) and the more classical species- or race-specific elicitors of $R$ genes. A similar mechanism could also explain why some plants show extremely frequent HR when confronted with members of a given viral genus, without having to postulate the existence of a specific $R$ gene for each HR-inducing virus. Such, for example, is observed in Gomphrena globosa, a plant known to possess a functional homolog of $R x$ (Goulden and Baulcombe 1993) and developing local lesions upon inoculation with over 20 potexviruses.

An interesting observation reported here is that, contrary to the situation observed with PVX (Jones 1985), the resistance against PepMV afforded by $R x$ is not durable and resistancebreaking isolates can be relatively easily selected upon mechanical inoculation of transgenic tomato plants expressing $R x$. Although a high level of variability was observed in the rate of detection of resistance-breaking variants between the individual experiments reported here, resistance-breaking variants were observed on a total of five of $62(8 \%)$ inoculated $R x$ plants (Table 1). Given the low number of plants generally involved in the experiments and the low overall frequency of emerging $R x$-breaking variants, a significant fraction of the interexperiment variability could simply be due to random stochastic variation. Similarly, although no $R x$-breaking variant was selected from isolate PV-0751, further efforts are clearly needed to establish whether $R x$ really affords a more durable resistance against this particular PepMV isolate.

$R x$-breaking variants could apparently be selected with a somewhat higher frequency from the mutant $R x 1179$ line (overall selection rate, $38 \%$ ). However, the number of plants involved again renders such a conclusion, at best, tentative. On the other hand, two elements seem to indicate that the outcome of the selection for resistance breaking in the two types of $R x$ plants is probably similar. First, of the four variants selected on $R x$ line 1179, three were observed to be stable upon further propagation on nonmutant, $R x$-expressing transgenics, indicating that they were already fully adapted. Second, mutations affecting identical amino acids were independently observed in different variants selected on either the wild-type or mutant $R x$ transgenics. In one case, a G232A (amino acid A78T) mutation was observed in variant nos. 8 (selected on $R x$ ) and 1179-1 (selected on $R x$ 1179) and in one of the sequences derived from variant $R x 10$ (selected on $R x$ ) whereas, in the other case, vari- ant nos. 22 (selected on $R x$ ) and 1179-5 (selected on $R x$ 1179) showed mutations affecting the glycine at position 99 of the capsid and changing it to an aspartate (G99D, variant 22) or to a serine (G99S, variant 1179-5).

Because the complete genomes of the $R x$-breaking variants have not been sequenced, and the effect of the CP mutations has not been verified using an infectious cDNA clone, the present data do not allow us to rule out formally the possibility that mutations outside the $\mathrm{CP}$ gene could be contributing to the observed resistance breaking. However, it is remarkable that all seven tested CP of resistance-breaking isolates had a greatly reduced or altogether abolished ability to induce an $R x$ mediated HR in transgenic $N$. benthamiana or tomato leaves. The fact that the severe symptoms induced by these variants on $R x$-expressing plants are not observed in wild-type plants, and therefore, are $R x$ mediated suggests, however, that some element of $R x$-mediated recognition is retained by all variant $C P$. Such is clearly observed to be the case in the $N$. benthamiana transient assay for variant nos. 1179-15-12 and 1179-1, and also, in the case of the tomato transient assay, for variant 11794; however, for the other variants, we cannot fully explain the difference observed between the behavior of the variant in the $R x$ tomato and that of its transiently expressed CP in the $R x N$. benthamiana plants. A similar situation has been reported by Farnham and Baulcombe (2006) in the case of PVX HB, which induces trailing necrosis in $R x$-expressing $N$. benthamiana plants whereas its $\mathrm{CP}$ does not induce HR upon transient expression in $R x$ plants.

Because many of the variant $\mathrm{CP}$ tested harbor more than one mutation, it is difficult to evaluate the contribution of individual mutations to the debilitation of their HR-inducing activity. However, mutations at positions 78 and 99, observed alone in variant nos. 8 and 1179-5, respectively, clearly have this effect. Mutations P105L and A114T observed in variant nos. 1179-1512 and 1179-4 in conjunction with other mutations that revert the PV-0632 sequence to that of other PepMV isolates controlled by $R x$ are also highly likely to be responsible for the loss of the $R x$-eliciting activity of the corresponding variants.

There are several possibilities to explain the lack of durability of the $R x$ resistance toward PepMV. One is that some additional host factors needed for the efficient activity of $R x$ might be missing or suboptimal in the transgenic tomato used, resulting in a decreased efficiency of the $R x$ resistance which could allow, in turn, the emergence of resistance-breaking isolates. It is difficult to eliminate this possibility but the observation that the original pepino isolate of PepMV was able to infect the USDA 41956 potato clone (Jones 1985), which is known to harbor a functional but uncharacterized $R x$ homolog (Querci et al. 1995), argues against this possibility. Another hypothesis could be that mutation toward partial or complete virulence could be easier for PepMV than for PVX. In the case of PVX, it is known that the resistance-breaking threonine-to-lysine mutation at position 121 of the CP also causes a severe loss in viral fitness (Goulden et al. 1993), so that at least one additional mutation at position 127 is required in order to recover a systemic infection-competent resistance-breaking isolate (Goulden et al. 1993). In contrast, in the case of PepMV, three of the seven analyzed resistance-breaking variants had singlepoint mutations in their CP (at positions 78 and 99), had CP with severely compromised or abolished $R x$-eliciting activity, and were able to mount a full systemic infection in their tomato hosts.

Further work, involving transient expression assays with mutated or deleted forms of the CP of PVX, PepMV, and other potexviruses should, in the future, allow better delineation of the conserved elements in the $\mathrm{CP}$ of these viruses recognized by $R x$ and could shed light on the mechanisms underlying the 
variations of durability of the resistance afforded by the $R x$ gene toward PVX and PepMV.

\section{MATERIALS AND METHODS}

\section{Viral isolates.}

Three PepMV isolates were obtained from the DSMZ (the German Collection of Microorganisms and Cell Cultures $\mathrm{GmbH}$, Braunschweig, Germany). Isolate PV-0554 is the original type isolate obtained from pepino (Jones et al. 1980) while isolates PV-0632 and PV-0751 are tomato isolates originating from Italy and Spain, respectively. A fourth isolate (1907) also obtained from tomato in Spain was a gift from M. C. Jorda (Universidad Politecnica de Valencia, Spain). All isolates were propagated under high-level containment greenhouse conditions by mechanical inoculation on either tomato plants (cv. Microtom) or $N$. benthamiana.

\section{Plant materials and inoculation assays.}

Transgenic tomato of cv. Microtom (Meissner et al. 1997) expressing the $R x$ gene under its own promoter was used in this study (A. Bendahmane, unpublished). The plants were obtained by Agrobacterium spp.-mediated transformation using the pSLJ9 recombinant plasmid (Bendahmane et al. 1999) which was initially used to obtain transgenic potato and $N$. benthamiana plants to validate the identification of $R x$ (Bendahmane et al. 1999). Absence of mutations in the introduced $R x$ gene has been verified by resequencing the $R x$ transgene following its introduction in all the transgenic lines used. Nontransgenic Microtom tomato plants were used as controls. In addition to the $R x$ transgenic Microtom line, an ethylmethane sulphonate mutant line derived from it and in which the $R x$-mediated HR phenotype is severely compromised (line $R x 1179$, MPD, TC and $\mathrm{AB}$ ) (unpublished) was also used in some experiments. All plants were inoculated at the two- to four-leaf stage by rub inoculation with infected plant homogenates $(1 \mathrm{~g}$ of plant material ground in $10 \mathrm{ml}$ of $25 \mathrm{mM} \mathrm{Na}_{2} \mathrm{HPO}_{4}$ supplemented with $0.2 \%$ diethyl dithiocarbomate) and Carborundum (400 mesh) used as an abrasive.

\section{Serological detection of PepMV.}

PepMV was detected by a standard double-antibody sandwich ELISA assay using commercial reagents (Plant Research International, Wageningen, The Netherlands). Alternatively, the same reagents were used in a membrane immunoprinting assay as described (Fakhfakh et al. 2001).

\section{PCR amplification and sequencing \\ of the PepMV capsid protein gene sequence comparisons.}

A pair of PCR primers allowing the precise amplification of the CP gene of PepMV was designed. The sequences of the two primers are downstream pepF2 primer (5' AAACAATTT AACTCAACTATG $3^{\prime}$ ) and pepR return primer (5' TGATTAA GTTTCGAGTGTTTA 3'). Reverse-transcriptase (RT)-PCR amplification of the $\mathrm{CP}$ gene was performed using $5 \mu \mathrm{l}$ of total nucleic acids samples obtained by a silica-capture method (Foissac et al. 2005) in a total volume of $50 \mu \mathrm{l}$ containing 10 $\mathrm{mM}$ Tris- $\mathrm{HCl}$ (pH 8.8), $1.5 \mathrm{mM} \mathrm{MgCl}_{2}, 50 \mathrm{mM} \mathrm{KCl}, 0.1 \%$ TritonX-100, $250 \mu \mathrm{M}$ dNTPs, $1 \mu \mathrm{M}$ each primer, $0.5 \mathrm{U}$ of AMLV (Amersham Biosciences, Buckinghamshire, U.K.), and $1 \mathrm{U}$ of $T a q$ DNA polymerase (Eurobio, Les Ulis, France). After an incubation at $42^{\circ} \mathrm{C}$ for $45 \mathrm{~min}$ and a denaturation step at $95^{\circ} \mathrm{C}$ for $5 \mathrm{~min}, 40$ cycles were applied, each consisting of $95^{\circ} \mathrm{C}$ for $30 \mathrm{~s}, 50^{\circ} \mathrm{C}$ for $30 \mathrm{~s}$, and $72^{\circ} \mathrm{C}$ for $1 \mathrm{~min}$. RT-PCR products were directly commercially sequenced (Cogenics, Meylan, France) on both strands. Sequences were manipulated and assembled using the suite of programs running on the "Se- quence Manipulation Suite" server. Database searches were performed using the National Center for Biotechnology Information (NCBI) BLAST server. Multiple sequences alignments were performed using the ClustalX program (Thompson et al. 1997).

\section{Transient expression of wild-type or mutated PepMV CP in $N$. benthamiana or tomato leaves by agroinfiltration.}

The PepMV CP genes amplified by PCR from the wild-type virus or from the various resistance-breaking variants were inserted between the $35 \mathrm{~S}$ promoter and terminator in the Agrobacterium binary vector pBin61 (Bendahmane et al. 1999). Oligonucleotide-directed mutagenesis was used to PCR amplify truncated forms of the PepMV CP which were similarly cloned in pBin61. Following full sequence verification, all constructs were transformed into Agrobacterium sp. strain $\mathrm{C} 58 \mathrm{C} 1$. The binary constructs were transiently expressed in wild-type and $R x$-expressing transgenic $N$. benthamiana or tomato leaves as previously described (Bendahmane et al. 2000). Verification of the expression of the various PepMV CP forms was performed by Western blot analysis of total protein extracts from agroinfiltrated leaves, using the same serological reagents as for the ELISA detection of PepMV.

\section{ACKNOWLEDGMENTS}

We thank S. Winter (DSMZ, Braunschweig, Germany) and M. C. Jorda (Universidad Politecnica de Valencia, Spain) for the generous gift of PepMV isolates, R. van der Vlugt (Plant Research International, Wageningen, The Netherlands) for PepMV-specific serological detection reagents, and D. Baulcombe for Rx MicroTom transgenic lines. The technical assistance of J. Peyrol and M. J. Dulucq with some experiments and of T. Mauduit and M. Roncoroni with the cultivation of the plants used is gratefully acknowledged, as well as comments of C. Kerlan (INRA Rennes, France) on PepMV-potato interactions.

\section{LITERATURE CITED}

Aguilar, J. M., Hernandez-Gallardo, M. D., Cenis, J. L., Lacasa, A., and Aranda M. A. 2002. Complete sequence of the Pepino mosaic virus RNA genome. Arch. Virol. 147:2009-2015.

Baratova, L. A., Grebenshchikov, N. I., Dobrov, E. N., Gedrovich, A. V., Kashirin, I. A., Shishkov, A. V., Efimov, A. V., Jarvekulg, L., Radavsky, Y. L., and Saarma, M. 1992a. The organization of Potato virus X coat proteins in virus particles studied by tritium planigraphy and model building. Virology 188:175-180.

Baratova, L. A., Grebenshchikov, N. I., Shishkov, A. V., Kashirin, I. A., Radavsky, J. L., Jarvekulg, L., and Saarma, M. 1992b. The topography of the surface of Potato virus X: Tritium planigraphy and immunological analysis. J. Gen. Virol. 73:229-235.

Baurès, I. 2008. Dissection génétique et moléculaire de la résistance par $R x$. Ph.D. thesis, Université Evry Val d'Essonne.

Baurès, I., Candresse, T., Leveau, A., Bendahmane, A., and Sturbois. B. 2008. The $R x$ gene confers resistance to a range of Potexviruses in transgenic Nicotiana plants. Mol. Plant-Microbe Interact. 21:11541164.

Bendahmane, A., Kohn, B. A., Dedi, C., and Baulcombe, D. C. 1995. The coat protein of Potato virus $X$ is a strain-specific elicitor of $R x 1$-mediated virus resistance in potato. Plant J. 8:933-941.

Bendahmane, A., Kanyuka, K., and Baulcombe, D. C. 1999. The Rx gene from potato controls separate virus resistance and cell death responses. Plant Cell 11:781-792.

Bendahmane, A., Querci, M., Kanyuka, K., and Baulcombe, D. C. 2000. Agrobacterium transient expression system as a tool for the isolation of disease resistance genes: Application to the $R x 2$ locus in potato. Plant J. 21:73-81.

Bent, A. F., and Mackey, D. 2007. Elicitors, effectors, and $R$ genes: The new paradigm and a lifetime supply of questions. Annu. Rev. Phytopathol. 45:399-436.

Boller, T., and Felix, G. 2009. A renaissance of elicitors: Perception of microbe-associated molecular patterns and danger signals by patternrecognition receptors. Annu. Rev. Plant Biol. 60:379-406.

Brunner, F., Rosahl, S., Lee, J., Rudd, J. J., Geiler, C., Kauppinen, S., Rasmussen, G., Scheel, D., and Nurnberger, T. 2002. Pep-13, a plant 
defense-inducing pathogen-associated pattern from Phytophthora transglutaminases. EMBO (Eur. Mol. Biol. Organ.) J. 21:6681-6688.

Chapman, S., Hills, G., Watts, J., and Baulcombe, D. C. 1992. Mutationa analysis of the coat protein gene of Potato virus $X$ : Effects on virion morphology and viral pathogenicity. Virology 191:223-230.

Chisholm, S. T., Coaker, G., Day, B., and Staskawicz, B. J. 2006. Hostmicrobe interactions: Shaping the evolution of the plant immune response. Cell 124:803-814.

Cockeram, G. 1970. Genetical studies on resistance to potato viruses X and Y. Heredity 25:309-348.

Cotillon, A. C., Girard, M., and Ducouret, S. 2002. Complete nucleotide sequence of the genomic RNA of a French isolate of Pepino mosaic virus (PepMV). Arch. Virol. 147:2231-2238.

Dolja, V. V., Boyko, V. P., Agranovsky, A. A., and Koonin, E. V. 1991. Phylogeny of capsid proteins of rod-shaped and filamentous RNA plant viruses: Two families with distinct patterns of sequence and probably structure conservation. Virology 184:79-86.

Ellis, J., Dodds, P., and Pryor, T. 2000. Structure, function and evolution of plant disease resistance genes. Curr. Opin. Plant Biol. 3:278-284.

Fakhfakh, H., Le Gall, O., Candresse, T., and Marrakchi, M. 2001. Analysis of the variability of Tunisian isolates of Lettuce mosaic virus using biological and molecular properties. J. Plant Pathol. 83:3-11.

Farnham, G., and Baulcombe, D. C. 2006. Artificial evolution extends the spectrum of viruses that are targeted by a disease-resistance gene from potato. Proc. Natl. Acad. Sci. U.S.A. 103:18828-18833.

Ferguson, G., and Shipp, L. 2002. New pests in Ontario greenhouse vegetables. Bull. OILB/SROP 25:69-72.

Foissac, X., Svanella-Dumas, L., Gentit, P., Dulucq, M. J., Marais, A., and Candresse, T. 2005. Polyvalent degenerate oligonucleotides reverse transcription-polymerase chain reaction: A polyvalent detection and characterization tool for trichoviruses, capilloviruses, and foveaviruses. Phytopathology 95:617-625.

French, C. J., Bouthillier, M., Bernardy, M., Ferguson, G., Sabourin, M. Johnson, R. C., Masters, C., Godkin, S., and Mumford, R. 2001. First report of Pepino mosaic virus in Canada and the United States. Plant Dis. 85:1121

Goulden, M. G., and Baulcombe, D. C. 1993. Functionally homologous host components recognize Potato virus $X$ in Gomphrena globosa and potato. Plant Cell 5:921-930.

Goulden, M. G., Kohm, B. A., Santa Cruz, S., Kavanagh, T. A., and Baulcombe, D. C. 1993. A feature of the coat protein of Potato virus $X$ affects both induced virus resistance in potato and viral fitness. Virology 197:293-302.

Hammond-Kosack, K. E., and Jones, J. D. 1997. Plant disease resistance genes. Annu. Rev. Plant Physiol. Plant Mol. Biol. 48:575-607.

Hanssen, I. M., Paeleman, A., Wittemans, L., Goen, K., Lievens, B. Bragard, C., Vanachter, A. C. R. C., and Thomma, B. P. H. J. 2008. Genetic characterization of Pepino mosaic virus isolates from Belgian greenhouse tomatoes reveals genetic recombination. Eur. J. Plant Pathol. 121:131-146.

Jones, R. A. C. 1985. Further studies of resistance-breaking strains of Potato virus $X$. Plant Pathol. 34:182-189.

Jones, R. A. C., Koenig, R., and Lesemann, D. E. 1980. Pepino mosaic virus, a new potexvirus from pepino (Solanum muricatum). Ann. Appl. Biol. 94:61-68.

Kavanagh, T., Goulden, M., Santa Cruz, S., Chapman, S., Barker, I., and Baulcombe, D. C. 1992. Molecular analysis of a resistance-breaking strain of Potato virus X. Virology 189:609-617.

Kohm, B. A., Goulden, M. G., Gilbert, J. E., Kavanagh, T. A., and Baulcombe, D. C. 1993. A Potato virus $X$ resistance gene mediates an induced, nonspecific resistance in protoplasts. Plant Cell 5:913-920.

Lopez, C., Soler, S., and Nuez, F. 2005. Comparison of the complete sequences of three different isolates of Pepino mosaic virus: Size variability of the TGBp3 protein between tomato and L. peruvianum isolates.
Arch. Virol. 150:619-627.

Maroon-Lango, C. J., Guaragna, M. A., Jordan, R. L., Hammond, J., Bandla, M., and Marquardt, S. K. 2005. Two unique US isolates of Pepino mosaic virus from a limited source of pooled tomato tissue are distinct from a third (European-like) US isolate. Arch. Virol. 150:11871201.

Martin, G. B., Bogdanove, A. J., and Sessa, G. 2003. Understanding the functions of plant disease resistance proteins. Annu. Rev. Plant. Biol. 54:23-61.

Martin, J., and Mousserion, C. 2002. Pomme de terre et mosaïque du pépino. Phytoma 552:26-28.

Meissner, R., Jacobson, Y., Melamed, S., Levyatuv, S., Shalev, G., Ashri, A., Elkind, Y., and Levy, A. 1997. A new model system for tomato genetics. Plant J. 12:1465-1472.

Moreira, A., Jones, R. A. C., and Fribourg, C. E. 1980. Properties of a resistance-breaking strain of Potato virus X. Ann. Appl. Biol. 95:93103.

Mumford, R. A., and Metcalfe, E. J. 2001. The partial sequencing of the genomic RNA of a UK isolate of Pepino mosaic virus and the comparison of the coat protein sequence with other isolates from Europe and Peru. Arch. Virol. 146:2455-2460.

Parker, J. E. 2003. Plant recognition of microbial patterns. Trends Plant Sci. 8:245-247.

Querci, M., van der Vlugt, R., Goldbach, R. W., and Salazar, L. F. 1993. RNA sequence of Potato virus $X$ strain HB. J. Gen. Virol. 74:22512255

Querci, M., Baulcombe, D. C., Goldbach, R. W., and Salazar, L. F. 1995. Analysis of the resistance-breaking determinants of Potato virus $X$ (PVX) strain $\mathrm{HB}$ on different potato genotypes expressing extreme resistance to PVX. Phytopathology 85:1003-1010.

Ritter, E., Debener, T., Barone, A., Salamini, F., and Gebhardt, C. 1991 RFLP mapping on potato chromosomes of two genes controlling extreme resistance to Potato virus X (PVX). Mol. Gen. Genet. 227:81-85.

Rommens, C. M., Salmeron, J. M., Oldroyd, G. E., and Staskawicz, B. J. 1995. Intergeneric transfer and functional expression of the tomato disease resistance gene Pto. Plant Cell 7:1537-1544.

Salomone, A., and Roggero, P. 2002. Host range, seed transmission and detection by ELISA and lateral flow of an Italian isolate of Pepino mosaic virus. J. Plant Pathol. 84:65-68.

Tai, T. H., Dahlbeck, D., Clark, E. T., Gajiwala, P., Pasion, R., Whalen, M. C., Stall, R. E., and Staskawicz, B. J. 1999. Expression of the Bs 2 pepper gene confers resistance to bacterial spot disease in tomato. Proc. Natl. Acad. Sci. U.S.A. 96:14153-14158.

Thompson, J. D., Gibson, T. J., Plewniak, F., Jeanmougin, F., and Higgins, D. G. 1997. The CLUSTAL_X windows interface: Flexible strategies for multiple sequence alignment aided by quality analysis tools. $\mathrm{Nu}$ cleic Acids Res. 25:4876-4882.

Tozzini, A. C., Ceriani, M. F., Saladrigas, M. V., and Hopp, H. E. 1991 Extreme resistance to infection by Potato virus $X$ in genotypes of wild tuber-bearing Solanum species. Potato Res. 34:317-324.

van der Vlugt, R. A. A., Stijger, C. C. M. M., Verhoeven, J. J. T., and Lesemann, D. E. 2000. First report of Pepino mosaic virus on tomato. Plant Dis. 84:103.

Whitham, S., McCormick, S., and Baker, B. 1996. The N gene of tobacco confers resistance to Tobacco mosaic virus in transgenic tomato. Proc. Natl. Acad. Sci. U.S.A. 93:8776-8781.

\section{AUTHOR-RECOMMENDED INTERNET RESOURCES}

Sequence Manipulation Suite server, version 2: www.bioinformatics.org/sms2

The National Center for Biotechnology Information BLAST server: www.ncbi.nlm.nih.gov/BLAST 